\title{
Diagnostic value of multimodal ultrasound imaging in differentiating benign and malignant TI-RADS category 4 nodules
}

\author{
Shufang Pei ${ }^{1,2} \cdot$ Shuzhen Cong ${ }^{1,2} \cdot$ Bin Zhang $^{3} \cdot$ Changhong Liang ${ }^{4} \cdot$ Lu Zhang $^{4} \cdot$ Juanjuan Liu $^{2} \cdot$ Yuping Guo $^{2}$. \\ Shuixing Zhang ${ }^{1,4}$
}

Received: 28 September 2018 / Accepted: 9 January 2019 / Published online: 1 March 2019

(c) The Author(s) 2019

\begin{abstract}
Background Differential diagnosis of benign and malignant thyroid imaging reporting and data system category 4 (TIRADS-4) nodules can be difficult using conventional ultrasound (US). This study aimed to evaluate whether multimodal ultrasound imaging can improve differentiation and characterization of benign and malignant TI-RADS-4 nodules.

Methods Multimodal ultrasound imaging, including US, superb microvascular imaging (SMI), and real-time elastography (RTE), were performed on 196 TI-RADS-4 nodules (78, benign; 118, malignant) in 170 consecutive patients. The sensitivity, specificity, accuracy, false negative rate (FNR), and false positive rate (FPR) of each single method and that of multimodal US imaging were determined by comparison with surgical pathology results.

Results The sensitivity, specificity, accuracy, FNR, and FPR for US were $65.25 \%, 69.23 \%, 66.84 \%, 34.75 \%, 30.77 \%$, respectively; for SMI were $77.97 \%, 93.59 \%, 84.18 \%, 22.03 \%, 6.41 \%$, respectively; RTE, $80.51 \%, 84.62 \%, 82.14 \%, 19.49 \%, 15.38 \%$; and for multimodal US imaging were $94.08 \%, 87.18 \%, 91.33 \%, 6.93 \%, 12.82 \%$, respectively. The areas under the received operating characteristic curve for US, SMI, RTE, and multimodal US imaging in evaluating benign and malignant TI-RADS-4 nodules were $67.2 \%, 84.40 \%, 86.60 \%$, and $95.50 \%$, respectively.

Conclusions The initial clinical results suggest that multimodal US imaging improves the diagnostic accuracy of TI-RADS-4 nodules and provides additional information for differentiating malignant and benign nodules.
\end{abstract}

Keywords Multimodal ultrasound Imaging $\cdot$ Superb microvascular imaging $\cdot$ Real-time elastography $\cdot$ Thyroid nodule

Shufang Pei, Shuzhen Cong, and Bin Zhang contributed equally to this work.

Shuixing Zhang

shui7515@126.com

1 The Second School of Clinical Medicine, Southern Medical University, Guangzhou, Guangdong,

People's Republic of China

2 Department of Ultrasound, Guangdong Academy of Medical Sciences/Guangdong General Hospital, Guangzhou, Guangdong, People's Republic of China

3 Department of Radiology, the First Affiliated Hospital of Jinan University, Guangzhou, Guangdong, People's Republic of China

4 Department of Radiology, Guangdong Academy of Medical Sciences/Guangdong General Hospital, No. 106 Zhongshan Er Road, Guangzhou 510080, Guangdong, People's Republic of China

\section{Introduction}

With the increased use of ultrasound (US) in medical practice, there has been an increase in the number of detected thyroid nodules, which are detected in about $20-67 \%$ of the general population [1-3]. It is, therefore, important to establish a standard method to accurately assess thyroid nodules. In May 2017, the American Academy of Radiology launched the Thyroid Imaging Reporting and Data System (TI-RADS) classification system [4], in which all thyroid nodules are divided into five categories: 1, 2, 3, 4, and 5. Categories 1-3 nodules are considered to be likely benign, and category 5 nodules are highly suspicious of being malignant. Typically, the nodule characteristics of categories 1-3 (benign) and category 5 (malignant) are obvious; therefore, the nodules can be easily diagnosed using conventional US $[5,6]$.

However, in contrast, differentiating benign and malignant TI-RADS category 4 nodules is difficult $[7,8]$. The ultrasonographic features of these thyroid nodules are 
complex [9-11], and the characteristics of conventional US images often overlap. The conventional ultrasound features of some nodules were shown to be benign, and the pathology of the surgery was confirmed to be malignant (Fig. 1); while the conventional ultrasound features of some nodules showed malignancy, and the pathology confirmed by surgery was benign (Fig. 2). Frequently, there are similarities and differences in the characteristics of these diseases, which can easily lead to misdiagnosis of patients and challenges for clinicians and patients. Therefore, it is particularly important to accurately diagnosis benign and malignant nodules. However, there is no relevant research concerning these issues.
Therefore, the purpose of this study was to evaluate the diagnostic performances of multimodal US imaging techniques in differentiating benign and malignant TI-RADS category 4 nodules. In an effort to improve the diagnostic accuracy for such nodules and facilitate clinical management, we investigated the following imaging techniques: conventional US, superb microvascular imaging (SMI), and real-time elastography (RTE).

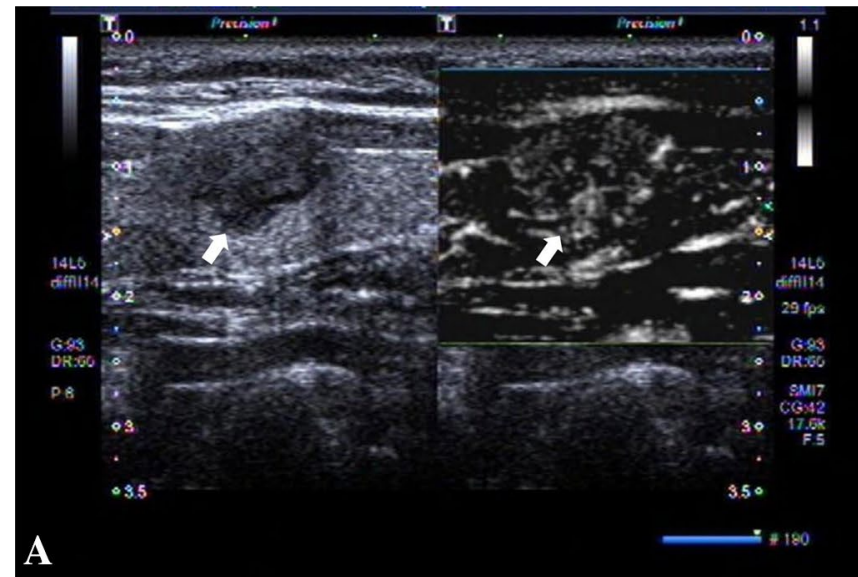

Fig. 1 Images in a 43-year-old woman who underwent routine. A $12 \mathrm{~mm}$ left thyroid nodule (arrows) with solid, hypo or isoechogenicity, well defined margin, wider than tall shape, no calcification was found at conventional ultrasound and assessed as benign, ACR score of 4 and classified as TI-RADS category 4 . A Type III was assigned

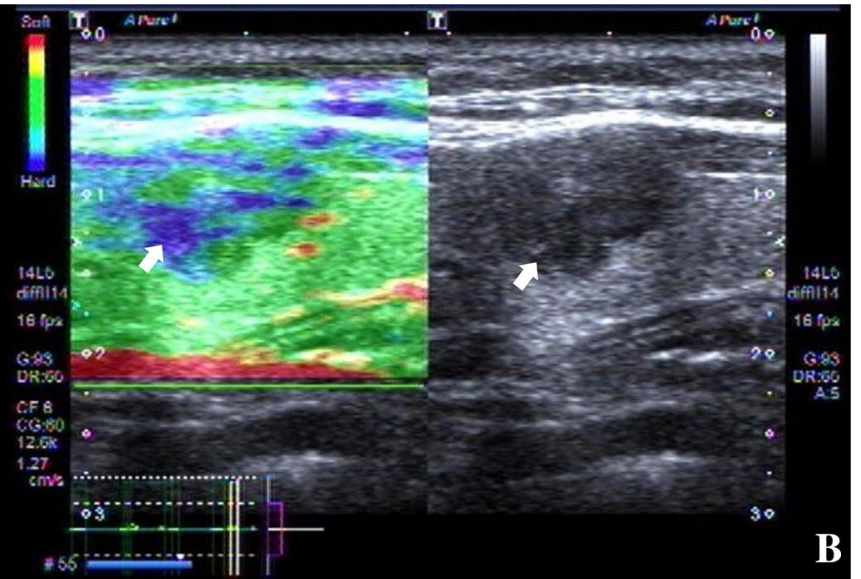

at superb microvascular imaging. B A score of 3 was assigned at realtime elastography. This nodules was assessed as malignant at multimodal ultrasound imaging. This thyroid nodule was diagnosed as papillary thyroid carcinoma at surgery

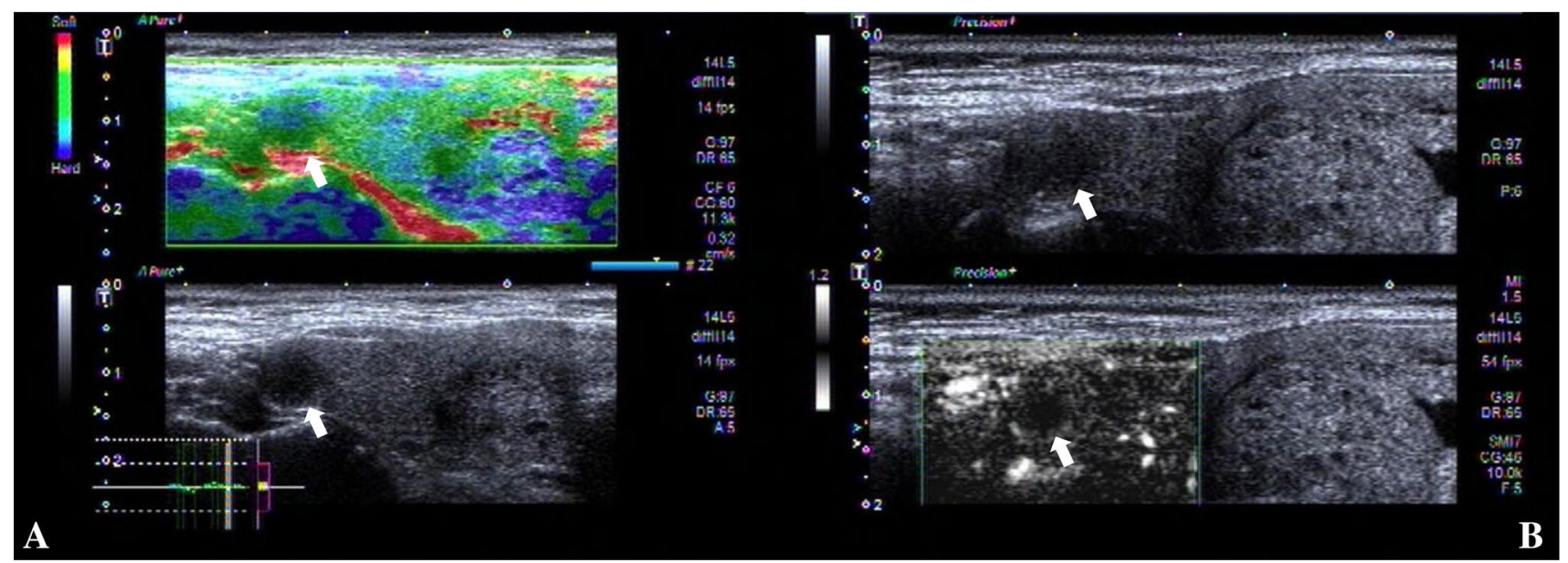

Fig. 2 Images in a 51-year-old man who underwent routine. A $7 \mathrm{~mm}$ left thyroid nodule (arrows) with solid, hypoechogenicity, irregularity margin, wider than tall shape, no calcification was found at conventional ultrasound and assessed as malignant, ACR score of 6 and classified as TI-RADS category 4. A Type II was assigned at superb microvascular imaging. B A score of 2 was assigned at real-time elastography. This nodules was assessed as benign at multimodal ultrasound imaging. This thyroid nodule was diagnosed as nodular goiter at surgery 


\section{Materials and methods}

The institutional review board approved this retrospective study and waived the requirement for patient approval or informed consent for the review of patient images and records.

This study was performed at our hospital from January 2016 to April 2018 and examined 698 thyroid nodules in 566 consecutive patients that were classified as TI-RADS category 4. Of these, 302 patients were excluded from the study because they did not undergo surgery in our hospital. A total of 316 thyroid nodules in 264 patients was diagnosed based on histopathological results from surgical excision or fine-needle aspiration. Of these, 120 cases thyroid nodules in 94 patients were excluded because elastography imaging could not be successfully performed and the results were obtained by fine needle puncture. Thyroid nodules that met the following criteria were included (Fig. 3): (a) those meeting the criteria for TI-RADS category 4; (b) all patients with complete data, including US indicators and pathological findings; (c) all nodules, in which RTE and SMI were successfully implemented; and (d) all thyroid nodules that were not subjected to minimally invasive surgery prior to US examination (such as puncture and ablation), because these operations can affect the evaluation of nodules by RTE and SMI. Finally, 196 solid thyroid nodules in 170 patients were included in this study. This group comprised 112 women and 58 men. Pathological results of all nodules were obtained through surgery $(n=196)$.

All patients, respectively, underwent three modes of US imaging with a high-frequency transducer (L14-5, Aplio 500) before surgery. The patients were maintained in the supine position, with the head tilted backward to fully expose the neck. The patients were asked to hold their breath and avoid swallowing. First, conventional US examination was performed to observe the ultrasonographic features of the thyroid nodules. According to the TI-RADS classification system, five features of each nodule were assessed, including components, echogenicity, shapes, margin, and calcification. Each feature was assigned a corresponding score, and then the nodules were assigned different TI-RADS classifications according to their total scores. Nodules with scores ranging from 4 to 6 were classified as TI-RADS category 4 , suggesting a moderate suspicion of malignancy.

\section{Superb microvascular imaging}

After conventional US examination, SMI was routinely performed by the same ultrasonographer who executed the conventional US. The SMI has two modes-monochrome and
Fig. 3 Inclusion criteria for the study. FNA fine-needle aspiration, $S M I$ superb microvascular imaging, RTE real-time elastography

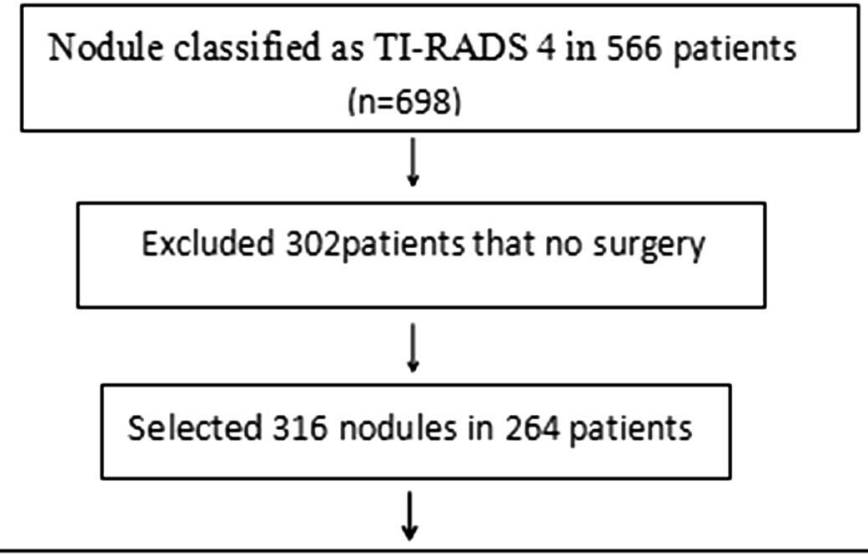

Excluded 120 nodules in 94 patients that SMI or RTE was not successfully
performed and the results were obtained by FNA

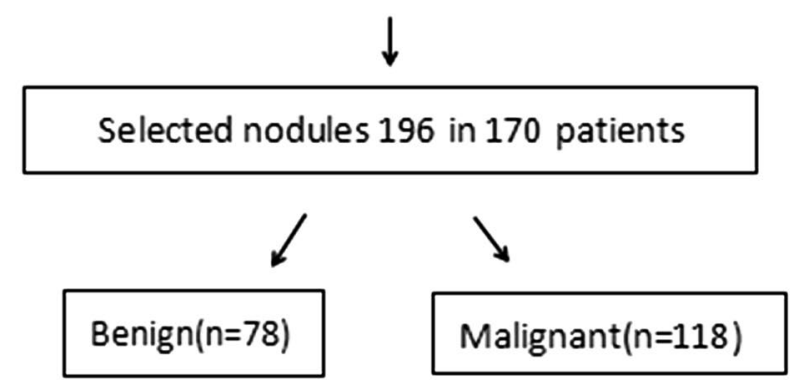


color. Because the former has greater sensitivity [12-14], we selected the monochrome SMI in our study. A double-screen function, which allowed simultaneous visualization of gray scale imaging and SMI, was used when vessels were located. During the imaging process, the patients were asked to breathe quietly and avoid swallowing, so as to reduce interference. In addition, the examiner used a multi-directional and multi-angle lateral motion probe to carefully observe the distribution of blood flow in the nodule. Once the most abundant and clearest section of the blood flow signal was identified, the nodule blood supply characteristics and blood flow distribution patterns were recorded. A semi-quantitative method was proposed by Kim et al. [15], in which vascularity was classified as four patterns: type I, no credible vascularity (defined as no perivascular or dotted perinodular flow on $<25 \%$ of the nodule's circumference and without any internal flow); type II, peripheral vascularity defined as surrounding $>25 \%$ of the nodule's circumference without any internal flow; type III, dominant internal blood flow (in the solid nodule, defined as an increase in the blood vessels which were curved, angled, spiral, or irregular); and type IV, mixed vascularity (defined as the presence of any intranodular flow and peripheral flow on $>25 \%$ of the nodule's circumference).

\section{Real-time elastography}

After SMI examination, real-time elastography was routinely performed by the same ultrasonographer who performed the US and SMI. All elastography images were obtained in longitudinal planes. During elastography, the probe was positioned perpendicular to the skin while applying pressure. Images were obtained by applying light repetitive compressions on the skin above the targeted thyroid nodule. A square region of interest (ROI), with the target nodule positioned at the center of the box, was set for elastography acquisition. The ROI needed to be set to include sufficient surrounding thyroid tissue because elasticity in this system is displayed relative to the average strain inside the ROI. Thus, the top of the ROI was set to include subcutaneous fat and the bottom of the ROI was set to include the pectoral muscle; the borders were set to more than $5 \mathrm{~mm}$ from the lesion's boundary $[16,17]$. The probe was maintained at the lesion site, while slight vibrations were induced. The pressure on the display device was controlled at an index of 3-4, with a clearly visible hardness at all levels of tissue, and continued for 3-4 s [18]. Malignant and benign thyroid nodules were diagnosed according to a 5-point scoring system with RTE based on the following color patterns: 0 points, for lesions with a cystic component showing red and blue or blue-green and red; 1 point, for lesions and surrounding tissue in an even green color; 2 points, for lesions in green and the surrounding area in blue; 3 points, for lesions in blue and green; and 4 points, for lesions completely colored in blue. For US elastography images, a score of 3 points and higher indicated a malignant nodule, while a score of less than 3 points suggested a benign nodule [19-21].

To avoid interobserver variability, two ultrasonographers (with 5 and 10 years of experience, respectively) who were blinded to any clinical information, recorded the sonographic features; if a disagreement existed between them, a third expert with 20 years of experience made the final determination.

\section{Statistical analysis}

The Student's $t$ test was used to compare patient age between the malignant and benign nodule groups. A $\chi^{2}$ test was used to analyze the differences in the distributions of conventional sonographic features, elastic score features, and vascular features between the groups. To investigate whether RTE, SMI, and the combination of the two methods could facilitate the diagnoses of TI-RADS category 4 thyroid nodules, the diagnostic value of any methods were compared using receiver operating characteristic curve (ROC) analysis. The $Z$ test was used to compare the area under the $\operatorname{ROC}\left(A_{\mathrm{z}}\right)$. Statistical significance was determined as $P<0.05$ (2-sided). Statistical analyses were performed using the SPSS version 20.0 statistical package (IBM Corporation, Armonk, NY, USA).

\section{Results}

\section{Pathological diagnosis}

The pathology results of 196 TI-RADS category 4 thyroid nodules identified 118 malignant thyroid nodules and 78 benign nodules; the malignant nodules comprised the following: papillary carcinoma $(n=107)$, medullary carcinoma $(n=4)$, follicular carcinoma $(n=4)$, and undifferentiated carcinoma $(n=3)$. The benign nodules comprised the following: thyroid adenoma $(n=12)$, nodular goiter $(n=60)$, and 6 others (subacute thyroiditis, $n=5$; Hashimoto's thyroiditis, $n=1)$.

\section{Features of conventional US, RTE, and SMI associated with benign and malignant TI-RADS category 4 thyroid nodules}

Conventional US features including solid, hypoechogenicity or marked echogenicity, an aspect ratio $\geq 1$, and microcalcifications were observed significantly more often in malignant nodules than in benign nodules (all $P<0.001$ ). Additionally, type III blood flow patterns (determined by SMI) and elastic scores of 3 and 4 were more significantly associated with 
Table 1 US, SMI and RTE

Features according to Malignant and Benign

\begin{tabular}{|c|c|c|c|}
\hline Feature & $\begin{array}{l}\text { No. of benign nodules } \\
(n=78)\end{array}$ & $\begin{array}{l}\text { No. of malignant nod- } \\
\text { ules }(n=118)\end{array}$ & $P$ value* \\
\hline Components & & & 0.002 \\
\hline \multicolumn{4}{|l|}{ Cystic $(n=0)$} \\
\hline \multicolumn{4}{|l|}{ Sponge-like $(n=0)$} \\
\hline Mixed $(n=21)$ & $15(71.4)$ & $6(28.6)$ & \\
\hline Solid $(n=175)$ & $63(36.0)$ & $112(64.0)$ & \\
\hline Echogenicity & & & $<0.001$ \\
\hline \multicolumn{4}{|l|}{ Anechogenicity $(n=0)$} \\
\hline Iso- or hyperechogenicity $(n=18)$ & $11(61.1)$ & $7(36.8)$ & \\
\hline Hypoechogenicity $(n=62)$ & $36(58.1)$ & $26(41.9)$ & \\
\hline Marked hypoechogenicity $(n=116)$ & $31(26.7)$ & $85(73.3)$ & \\
\hline \multicolumn{3}{|l|}{ Shape } & 0.011 \\
\hline Wider than tall $(n=114)$ & $54(47.4)$ & $60(52.6)$ & \\
\hline Taller than wide $(n=82)$ & $24(29.3)$ & $58(70.7)$ & \\
\hline \multicolumn{3}{|l|}{ Margin } & 0.873 \\
\hline Well defined $(n=30)$ & $14(46.7)$ & $16(53.3)$ & \\
\hline Poorly defined $(n=148)$ & $57(38.5)$ & $91(61.5)$ & \\
\hline Irregularity or lobuling $(n=5)$ & $2(40.0)$ & $3(60.0)$ & \\
\hline Extracapsular spread $(n=17)$ & $5(38.5)$ & $8(61.5)$ & \\
\hline \multicolumn{3}{|l|}{ Calcification } & $<0.001$ \\
\hline No calcification $(n=87)$ & $44(50.6)$ & $43(49.4)$ & \\
\hline Macrocalcification $(n=30)$ & $19(63.3)$ & $11(36.7)$ & \\
\hline Peripheral calcification $(n=9)$ & $9(100)$ & $0(0)$ & \\
\hline Microcalcification $(n=57)$ & $4(7.0)$ & $53(93.0)$ & \\
\hline Macro + Microcalcification $(n=13)$ & $2(15.4)$ & $11(84.6)$ & \\
\hline \multicolumn{3}{|l|}{ SMI } & $<0.001$ \\
\hline $\mathrm{I}(n=27)$ & $22(81.5)$ & $5(18.5)$ & \\
\hline II $(n=49)$ & $44(89.8)$ & $5(10.2)$ & \\
\hline III $(n=97)$ & $5(5.2)$ & $92(94.8)$ & \\
\hline IV $(n=23)$ & $7(30.4)$ & $16(69.6)$ & \\
\hline \multicolumn{3}{|l|}{ RTE } & $<0.001$ \\
\hline \multicolumn{4}{|l|}{$0(n=0)$} \\
\hline $1(n=29)$ & $29(100)$ & $0(0)$ & \\
\hline $2(n=60)$ & $37(61.7)$ & $23(38.3)$ & \\
\hline $3(n=101)$ & $12(11.9)$ & $89(88.1)$ & \\
\hline $4(n=6)$ & $0(0)$ & $6(100)$ & \\
\hline
\end{tabular}

Unless otherwise indicated, data are numbers of nodules, and numbers in parentheses are percentages $S M I$ superb microvascular imaging, RTE real-time elastography

$* P$ values were calculated using generalized estimating equation analysis malignant nodules than with benign nodules (all $P<0.001$ ). The details were presented in Table 1 .

\section{Diagnostic performances of US, RTE, and SMI- which were applied alone and in combination-in evaluating TI-RADS category 4 nodules}

In regard to the 196 nodules, the sensitivities, specificities, and accuracies of RTE and SMI were significantly higher than those of US. With regards to diagnosing TI-RADS category
4 nodules, RTE had a higher sensitivity than that of SMI, but the false positive rate of RTE was higher than that of SMI (Table 2). The accuracy of the combination of the three methods was significantly higher than that of each of the three methods separately (Table 2; Fig. 4). 
Table 2 Diagnostic performance of US, RTE and SMI in 196 Nodules in 170 Patients

\begin{tabular}{lllllr}
\hline Methods & Sensitivity $(\%)$ & Specificity $(\%)$ & Accuracy $(\%)$ & FNR(\%) & FPR(\%) \\
\hline US & 65.25 & 69.23 & 66.84 & 34.75 & 30.77 \\
RTE & 80.51 & 84.62 & 82.14 & 19.49 & 15.38 \\
SMI & 77.97 & 93.59 & 84.18 & 22.03 & 6.41 \\
Multimodal & 94.08 & 87.18 & 91.33 & 6.93 & 12.82 \\
\hline
\end{tabular}

$F N R$ false negative rate, $F P R$ false positive rate, $S M I$ superb microvascular imaging, $R T E$ real-time elastography, US conventional ultrasound
Fig. 4 ROC curves of US, RTE and SMI in evaluating benign and malignant TI-RADS4 nodules. SM I superb microvascular imaging, RTE real-time elastography, US conventional ultrasound

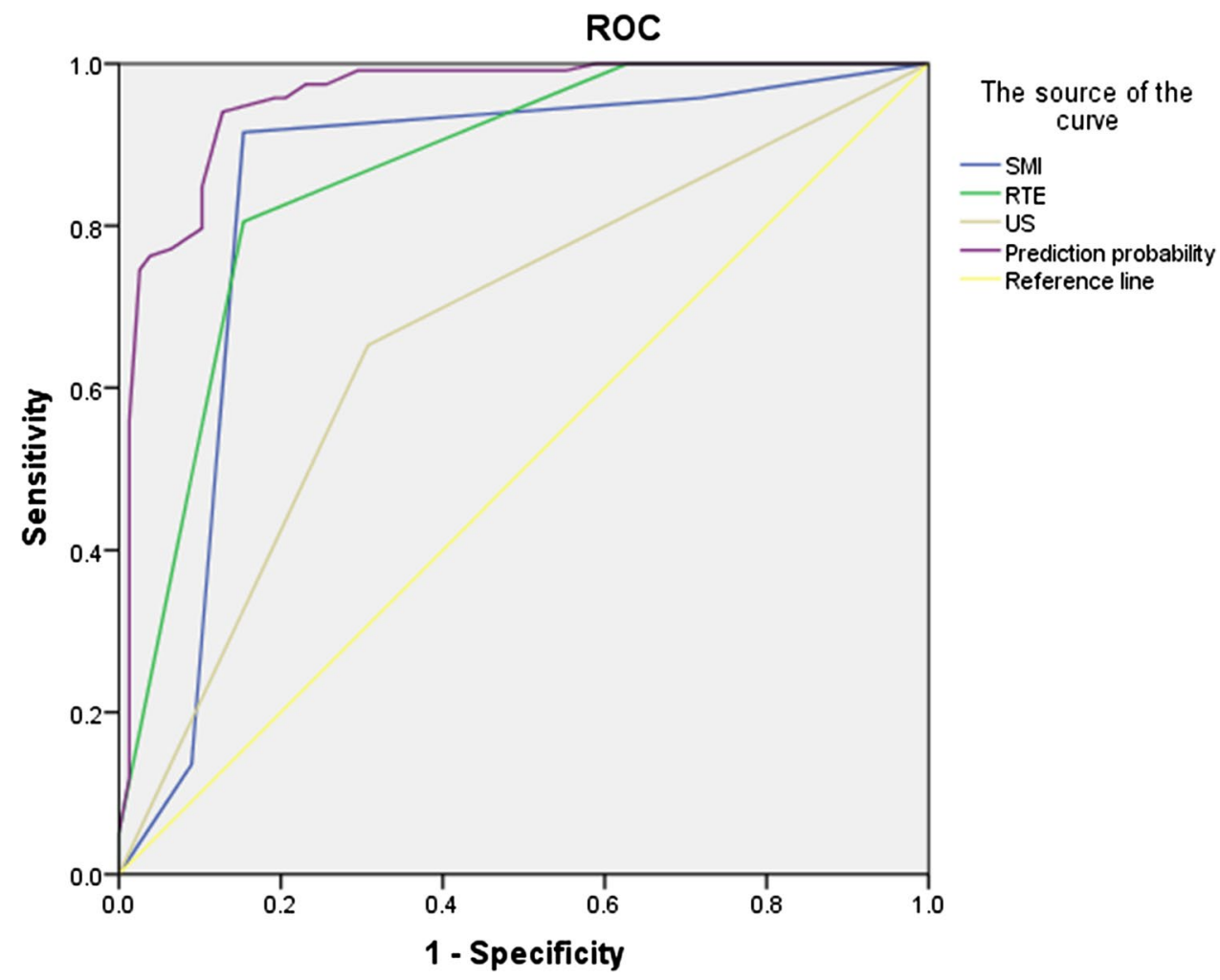

\section{Discussion}

In our study, the sensitivity, specificity, and accuracy of US in the diagnosis of TI-RADS category 4 nodules were $65.25 \%, 69.23 \%$, and $66.84 \%$, respectively. Compared with previous studies, our results were lower than those of US in the diagnosis of benign and malignant all thyroid nodules [18]: this may be related to the nodules we studied. In the past, conventional US was used to evaluate all thyroid nodules, but the nodules of our study were classified as TI-RADS category 4 . The benign and malignant characteristic of this type of nodules were often not obvious, and it was difficult to determine if they were benign or malignant by US alone. Therefore, it is necessary to establish a more effective method to accurately diagnose benign and malignant nodules of this type.
In this study, we evaluated TI-RADS 4 nodules using RTE. According to previous studies, a score of 3 was used as a diagnostic cut-off value. When the nodule elastic score was greater than or equal to 3 , it was classified as malignant; those with a score less than 3 , were classified as benign. Our results confirmed, that for the nodules of TI-RADS category 4 (which were difficult to identify using conventional US), the sensitivity, specificity, and accuracy of the RTE were $80.51 \%, 84.62 \%$, and $82.14 \%$, respectively. These results are comparable to those of Magri et al. [16, 17, 22, 23], who reported a sensitivity between 84.40 and $89.10 \%$ and a specificity between 70.00 and $85.30 \%$. Our results showed that RTE has a higher sensitivity, specificity and accuracy than US in diagnosing TI-RADS 4 nodules, and the difference between the two methods was statistically significant $(P<0.05)$. However, it was clear that RTE had a relatively 
high false positive rate in diagnosing TI-RADS category 4 nodules. This may be attributed to multiple reasons [24-28]. For example, this study included some nodules with peripheral circular calcifications or internal semi-arc calcifications, in which the presence of calcifications may have increased the elasticity score. Additionally, to comprehensively evaluate the nodule, the other US features should be combined with that of RTE. On the other hand, some of the nodules were characterized by a mixed echo pattern. This included hypoechoic and slightly strong echo patterns and irregular forms, which are often caused by long-term fibrosis of the nodules, which also leads to increased elasticity scores.

When SMI was used to evaluate TI-RADS 4 nodules, it showed good diagnostic values. In terms of diagnosing benign and malignant TI-RADS 4 nodules, the sensitivity, specificity, and accuracy of SMI were $77.97 \%, 93.59 \%$, and $84.18 \%$, respectively. These results are consistent with those from a study by Kong et al. [29], who reported a sensitivity of $75.9 \%$, and a specificity of $91.2 \%$; other studies reported a sensitivity of $78.91 \%$ and a specificity of $85.1 \%$ [30]. Compared with RTE, SMI had a lower false positive rate. A possible reason for this is that the existence of ring and arc-calcifications may have led to a higher elasticity score and an inaccurate assessment. However, SMI can accurately assess the distribution pattern of blood flow within the nodule under these circumstances, thereby avoiding the effect of macro-calcifications.

Our results suggest that using both RTE and SMI, in conjunction with US, is a reliable method to differentiate between benign and malignant TI-RADS 4 nodules. When US was combined with the two other methods, the sensitivity, specificity, and accuracy in diagnosing TI-RADS 4 nodules were $94.08 \%, 87.18 \%$, and $91.33 \%$, respectively. These results were better than that of US, RTE, or SMI alone, and the difference was statistically significant $(P<0.05)$. Compared with US, RTE, and SMI, the accuracy of multimodal US imaging was increased, and the false negative rate decreased in terms of diagnosing benign and malignant TIRADS 4 nodules. At the same time, the area under the ROC curve showed that multimodal US imaging was superior to the use of US, SMI, or RTE alone in the evaluation of benign and malignant TI-RADS 4 nodules $(P<0.001)$ (Fig. 4).

The above results may be related to the following reasons. First, in regard to the combination of the three methods, SMI helped compensate for the disadvantages of RTE in situations of calcification, depth, and trachea. This is because during elastography imaging, if the location was too deep, too closes to the trachea, or if the nodules presented with peripheral annular calcifications, those factors affected the elasticity score. On the other hand, for some smaller malignant lesions, the blood distribution pattern of SMI may show a type I or II pattern, leading to a false negative result. However, RTE was able to obtain information on the "hardness" of nodules through an elasticity score and achieve accurate diagnoses.

This study did have some limitations. First, the number of nodules we studied was small. Second, the pathological types of the nodules were relatively simple. The malignant nodules comprised primarily papillary carcinoma, with only four medullary carcinomas, four follicular carcinomas, and three undifferentiated carcinomas. Third, the assessments of RTE and SMI were not grouped according to nodular size. In future studies, we plan to collect more data, classify nodules according to size, and evaluate the diagnostic performances of SMI and RTE within the different groups of nodules.

The results of this study suggest that the application of RTE and SMI may help compensate in areas in which conventional US may be deficient in assessing the TI-RADS category 4 nodules; thus, multimodal US imaging — using the three methods-may provide more comprehensive information regarding the nodules, facilitating more accurate diagnoses. In conclusion, multimodal US imaging is beneficial in assessing TI-RADS category 4 nodules.

Acknowledgements We acknowledge financial support from the Medical Science and Technology Foundation of Guangdong Province $(\mathrm{CN})$ (A2018348) and the Guangzhou Science, Technology and Innovation Commission (CN) (201804010105).

Author contributions statement SP: planning study design and conducting study, data collection, writing of the manuscript, and final approval of the manuscript. SC: data collection, writing of the manuscript, and final approval of the manuscript. BZ: planning study design and conducting study, data analysis, manuscript revise and editing, and final approval of the manuscript. CL: final approval of the manuscript. LZ, JL and YG: data collection, writing of the manuscript, and final approval of the manuscript. SZ: data interpretation and data review/approval, and editing of the manuscript, and final approval of the manuscript.

\section{Compliance with ethical standards}

Conflict of interest The authors declare that they have no conflict of interest.

Ethical approval All procedures performed in studies involving human participants were in accordance with the ethical standards of the Institutional Review Board and with the 1964 Helsinki declaration and its later amendments or comparable ethical standards.

Informed consent Our Institutional Review Board waived the requirement to obtain informed consent from patients.

Open Access This article is distributed under the terms of the Creative Commons Attribution 4.0 International License (http://creativeco mmons.org/licenses/by/4.0/), which permits unrestricted use, distribution, and reproduction in any medium, provided you give appropriate credit to the original author(s) and the source, provide a link to the Creative Commons license, and indicate if changes were made. 


\section{References}

1. Ross DS (2002) Nonpalpable thyroid nodules-managing an epidemic. J Clin Endocrinol Metab 87(5):1938-1940

2. Hu X, Liu Y, Qian L (2017) Diagnostic potential of real-time elastography (RTE) and shear wave elastography (SWE) to differentiate benign and malignant thyroid nodules. Medicine (Baltimore) 96(43): 8282

3. Liu Y, Wu H, Zhou Q et al (2002) Diagnostic value of conventional ultrasonography combined with contrast-enhanced ultrasonography in thyroid imaging reporting and data system (TI-RADS) 3 and 4 thyroid micronodules. Med Sci Monit 22:3086-3094

4. Tessler FN, Middleton WD, Grant EG et al (2017) ACR Thyroid Imaging, Reporting and Data System (TI-RADS): White Paper of the ACR TI-RADS Committee. J Am Coll Radiol 14(5):587-595

5. Ulisse S, Bosco D, Nardi F et al (2017) Thyroid imaging reporting and data system score combined with the new italian classification for thyroid cytology improves the clinical management of indeterminate nodules. Int J Endocrinol 2017:1-8

6. Ko SY, Lee HS, Kim EK et al (2014) Application of the thyroid imaging reporting and data system in thyroid ultrasonography interpretation by less experienced physicians. Ultrasonography 33(1):49-57

7. Zhang Y, Zhou P, Tian SM et al (2017) Usefulness of combined use of contrast-enhanced ultrasound and TI-RADS classification for the differentiation of benign from malignant lesions of thyroid nodules. Eur Radiol 27(4):1527-1536

8. Xu T, Gu JY, Ye XH et al (2017) Thyroid nodule sizes influence the diagnostic performance of TIRADS and ultrasound patterns of 2015 ATA guidelines: a multicenter retrospective study. Sci Rep 7:43183

9. Du YR, Ji CL, Wu Y et al (2018) Combination of ultrasound elastography with TI-RADS in the diagnosis of small thyroid nodules $(</=10 \mathrm{~mm})$ : A new method to increase the diagnostic performance. Eur J Radiol 109:33-40

10. Shangguan R, Hu YP, Huang J et al (2018) Association Between BRAF(V600E) mutation and the American College of Radiology thyroid imaging, reporting and data system in solitary papillary thyroid carcinoma. Acad Radiol 26(2):154-160

11. Tessler FN, Middleton WD, Grant EG (2018) Thyroid imaging reporting and data system (TI-RADS): a user's guide. Radiology 287(3):1082

12. Hasegawa J, Suzuki N (2016) SMI for imaging of placental infarction. Placenta 47:96-98

13. Wu L, Yen HH, Soon MS (2015) Spoke-wheel sign of focal nodular hyperplasia revealed by superb micro-vascular ultrasound imaging. QJM 108(8):669-670

14. Kong J, Li JC, Wang HY et al (2017) Role of superb micro-vascular imaging in the preoperative evaluation of thyroid nodules: comparison with power Doppler flow imaging. J Ultrasound Med 36(7):1329-1337

15. Kim DW, Jung SJ, Eom JW, Kang T (2013) Color Doppler features of solid, round, isoechoic thyroid nodules without malignant sonographic features: a prospective cytopathological study. Thyroid 23(4):472-476
16. Ko SY, Kim E, Sung JM et al (2014) Diagnostic performance of ultrasound and ultrasound elastography with respect to physician experience. Ultrasound Med Biol 40(5):854-863

17. Wang H, Zhao L, Xin X et al (2014) Diagnostic value of elastosonography for thyroid microcarcinoma. Ultrasonics 54(7):1945-1949

18. Shuzhen C (2012) Comparison analysis between conventional ultrasonography and ultrasound elastography of thyroid nodules. Eur J Radiol 81(8):1806-1811

19. Zhang Y, Xu T, Gong H et al (2016) Application of high-resolution ultrasound, real-time elastography, and contrast-enhanced ultrasound in differentiating solid thyroid nodules. Medicine (Baltimore) 95(45):e5329

20. Tian W, Hao S, Gao B et al (2016) Comparing the diagnostic accuracy of RTE and SWE in differentiating malignant thyroid nodules from benign ones: a meta-analysis. Cell Physiol Biochem 39(6):2451-2463

21. Sui X, Liu HJ, Jia HL et al (2016) Contrast-enhanced ultrasound and real-time elastography in the differential diagnosis of malignant and benign thyroid nodules. Exp Ther Med 12(2):783-91

22. Magri F, Chytiris S, Zerbini F et al (2015) Maximal stiffness evaluation by real-time ultrasound elastography, an improved tool for the differential diagnosis of thyroid nodules. Endocr Pract 21(5):474-481

23. Liu B, Xie X, Liang J et al (2014) Shear wave elastography versus real-time elastography on evaluation thyroid nodules: a preliminary study. Eur J Radiol 83(7):1135-1143

24. Lippolis PV, Tognini S, Materazzi G et al (2011) Is elastography actually useful in the presurgical selection of thyroid nodules with indeterminate cytology? J Clin Endocrinol Metab 96(11):E1826-E1830

25. Bojunga J, Herrmann E, Meyer G et al (2010) Real-time elastography for the differentiation of benign and malignant thyroid nodules: a meta-analysis. Thyroid 20(10):1145-1150

26. Merino S, Arrazola J, Cárdenas A et al (2011) Utility and interobserver agreement of ultrasound elastography in the detection of malignant thyroid nodules in clinical care. AJNR Am J Neuroradiol 32(11):2142-2148

27. He YP, Xu HX, Zhao CK et al (2017) Cytologically indeterminate thyroid nodules: increased diagnostic performance with combination of US TI-RADS and a new scoring system. Sci Rep 7(1):6906

28. Rago T, Santini F, Scutari M et al (2007) Elastography: new developments in ultrasound for predicting malignancy in thyroid nodules. J Clin Endocrinol Metab 92(8):2917-2922

29. Kong J, Li J, Wang H et al (2017) Role of superb micro-vascular imaging in the preoperative evaluation of thyroid nodules: comparison with power doppler flow imaging. J Ultrasound Med 36(7):1329-1337

30. Lu R, Meng Y, Zhang Y et al (2017) Superb microvascular imaging (SMI) compared with conventional ultrasound for evaluating thyroid nodules. BMC Med Imaging 17(1):65

Publisher's Note Springer Nature remains neutral with regard to jurisdictional claims in published maps and institutional affiliations. 